The Use Of Scanning Probe Microscopy To Investigate Crystal-Fluid Interfaces

C. A. Orme, J. L. Giocondi

April 23, 2007

Perspectives on Inorganic, Organic, and Biological Crystal Growth: from Fundamentals to Applications 
This document was prepared as an account of work sponsored by an agency of the United States government. Neither the United States government nor Lawrence Livermore National Security, LLC, nor any of their employees makes any warranty, expressed or implied, or assumes any legal liability or responsibility for the accuracy, completeness, or usefulness of any information, apparatus, product, or process disclosed, or represents that its use would not infringe privately owned rights. Reference herein to any specific commercial product, process, or service by trade name, trademark, manufacturer, or otherwise does not necessarily constitute or imply its endorsement, recommendation, or favoring by the United States government or Lawrence Livermore National Security, LLC. The views and opinions of authors expressed herein do not necessarily state or reflect those of the United States government or Lawrence Livermore National Security, LLC, and shall not be used for advertising or product endorsement purposes. 


\title{
The Use Of Scanning Probe Microscopy To Investigate Crystal-Fluid Interfaces
}

\author{
Christine A. Orme and Jennifer L. Giocondi \\ Chemistry, Materials and Life Sciences Directorate, Lawrence Livermore National Laboratory, \\ 7000 East Ave., Mailstop L-350, Livermore, CA 94550
}

\begin{abstract}
Over the past decade there has been a natural drive to extend the investigation of dynamic surfaces in fluid environments to higher resolution characterization tools. Various aspects of solution crystal growth have been directly visualized for the first time. These include island nucleation and growth using transmission electron microscopy and scanning tunneling microscopy; elemental step motion using scanning probe microscopy; and the time evolution of interfacial atomic structure using various diffraction techniques. In this lecture we will discuss the use of one such in situ method, scanning probe microscopy, as a means of measuring surface dynamics during crystal growth and dissolution. We will cover both practical aspects of imaging such as environmental control, fluid flow, and electrochemical manipulation, as well as the types of physical measurements that can be made. Measurements such as step motion, critical lengths, nucleation density, and step fluctuations, will be put in context of the information they provide about mechanistic processes at surfaces using examples from metal and mineral crystal growth.
\end{abstract}

Keywords: Crystal growth, scanning probe microscopy, SPM, in situ imaging, surface dynamics, step kinetics, critical length.

PACS: 81.10.Dn, 68.37.Ps, 68.08.-p

\section{INTRODUCTION}

The dialogue surrounding crystal growth depends to a great degree on what we are able to measure. And for this reason the development of the field has followed a natural progression trending towards smaller length scales and shorter timescales as facets, then steps and islands, and ultimately adatoms and kinks have come literally into view (Fig. 1).

Early crystal growth science focused on the faceted macroscopic state of solution grown crystals. At this stage imaging consisted of optical micrographs of the entire crystal and kinetic measurements were of bulk growth rates. In keeping with observations, the habit (distribution of facets) and the thermodynamics of phase transition dominated this stage of crystal growth science[1]. Moving down in scale, step trains and (widely spaced) spiral steps[2] became measurable during the first half of the 1900s using interferometry and step decoration techniques. These images of spirals and growth hillocks inspired the development of BCF[3] theory and its many extensions[4, 5]. Interferometry also improved the resolution of kinetic measurements and could resolve step train motions of approximately 10 atomic steps.

The next big advances in crystal growth physics came predominately from observations of vapor deposited films. The advent of ultra-high vacuum (UHV) techniques to deposit thin metal and semiconductor films allowed a much higher degree 
of control over both growth mode and impurity concentrations as compared to solution growth. When these modern thin film deposition methods were coupled with techniques capable of atomic (or near atomic) resolution such as Scanning Tunneling Microscopy (STM) [6, 7], Transmission Electron Microscopy (TEM), Focused Ion Microscopy[8, 9] (FIM), and Low Energy Electron microscopy (LEEM)[10-12], concepts such as adatoms, kinks, kink densities, step fluctuations, step distributions, island size distributions, island spacing distributions, and surface reconstructions, all became observables whose relationship to growth parameters could be directly tested.

However, most microscopes cannot image during deposition and instead growth is evaluated using the "stop and look" approach where the evolution of the surface configuration is quenched by turning off the flux and quickly lowering the temperature. Kinetics are indirectly inferred from snapshots of surfaces before and after evolution. Variable temperature instruments opened the possibility of imaging dynamic processes but again of surfaces either in equilibrium or evolving towards equilibrium rather than during growth. Nevertheless, this style of experiment has provided (and continues to provide) a wealth of exciting data that forms most of what is known quantitatively of activation barriers and surface diffusion $[13,14]$. With a few exceptions, it is only in the past ten years that true in situ growth measurements have been used to complement variable temperature studies in developing and testing the fundamentals of materials assembly.

There are only a few instruments capable of true in situ imaging. For vapor deposition these are LEEM, STM[15], and TEM[16] whereas for solution crystal growth these are fluid cell TEMs[17, 18] and various flavors of scanning probe microscopes. Except for SPMs, all of these are specialized or unique instruments. In this sense SPMs represent in situ crystal growth opportunities for the people! And in the remainder of the chapter we will concentrate on their role in examining solution-based crystal growth.

Current commercial SPMs are best suited for measuring features with lateral dimensions in the nanometers to microns range, heights less than a few microns, and dynamics that evolve on timescales of seconds to minutes. This makes them ideally suited (almost out of the box) for studying the atomic step motion of a broad class of solution grown materials from minerals to organic solids. They are also well suited for following the nucleation and growth of many metals and oxides although in these cases often the single atomic steps are not resolvable.

Since the first SPM studies of solution crystal growth in 1988[19], many fundamental aspects of crystal growth have been examined directly for the first time. This has allowed theoretical predictions to be scrutinized with much greater detail and in many cases has presented difficulties with current models of crystal growth[20, 21].

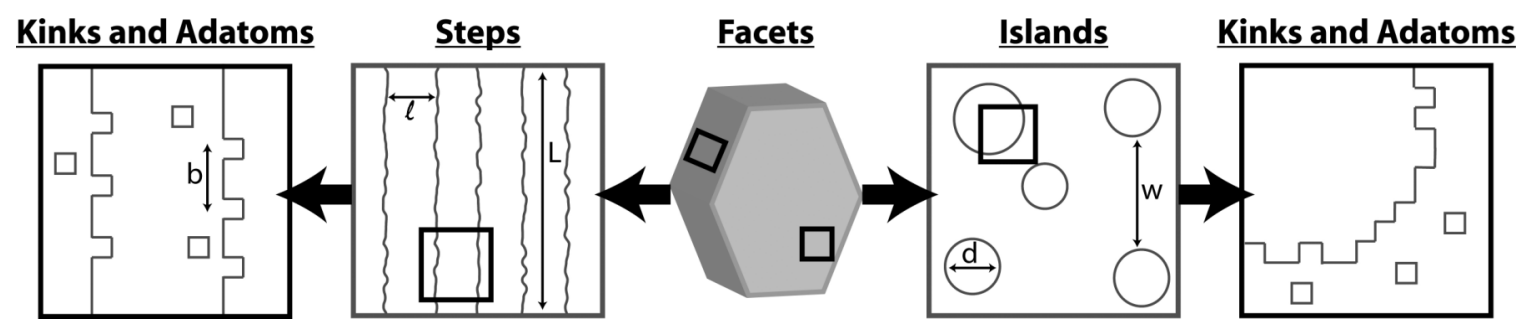

FIGURE 1. A illustration of how crystal growth science has evolved from the study of macroscopic facets to the atomic scale of kinks and adatoms. 
In this chapter we begin with the basic operating principles of the atomic force microscope along with some practical aspects of imaging in a fluid environments. The controlling experimental parameters ("the knobs") of solution crystal growth are largely determined by the solution state and include the supersaturation, the $\mathrm{pH}$, the ionic strength, and the ratios of various active species. Other parameters available to the experimentalist are the temperature, the solution flow rate, and, for electrochemical deposition, the current or voltage. Because knowledge of these parameters is essential for quantitative measurements, we will briefly discuss solution speciation. The quantitative measures depend largely on the substrate materials and the intent of the experiment but several common measurements are step velocities, critical lengths, and morphology.

\section{SPM BASICS}

The atomic force microscope[22] (or more generally the scanning probe microscope) was invented in 1986 only a few years after the scanning tunneling microscope astonished the scientific world by obtaining atomic resolution images of the $7 \times 7$ reconstruction of silicon[23]. The STM operates by measuring the tunneling current between a metallic tip and a conducting substrate. To expand this imaging capability to non-conducting materials the atomic force microscope (AFM) instead measures the interaction force between a probe and a surface. The operating principles of scanning probe microscopes have been discussed at length in many review articles and books[24]. Thus we will only briefly cover basic components and operating modes and instead we will focus on the challenges as well as some advantages associated with imaging in a fluid phase.

The basic components of SPMs are the probe, scanner, detector, and feedback system (Fig. 2). The SPM relies on tracking the sample surface using the interatomic forces between the sample and a probe[25]. This probe is mounted on the free end of a cantilever and the radius of curvature of the probe apex, which is typically on the order of $10 \mathrm{~nm}$, determines the resolution. The scanner is made of a piezoelectric ceramic that changes shape under an applied voltage and is used to translate the relative position between the probe and the sample across an $x-y$ grid. As the tip is rastered over the sample, the cantilever bends in response to changes in topography. These cantilever deflections are monitored by the change in position of an optical beam that is bounced off the back of the cantilever onto a position sensitive photo detector. This change in beam position is used as input into a feedback system that adjusts the tip-sample (z) distance and is used to construct a topographic image of the surface (Fig. 2).

Topography can be measured in one of three ways: contact, intermittent contact, and non-contact. The differences in these modes arise from both the type of force experienced between the probe and the sample and the vibration of the cantilever. As the distance between the probe and the sample is decreased the atoms in the two materials begin to interact. Initially this interaction is attractive, as shown in the bottom left of Fig. 3, but as the atoms get closer electrostatic repulsion between the electron clouds dominate. This repulsive regime is where contact mode is performed. The repulsive force between the atoms of the probe and sample is greater than the force applied by the cantilever thus causing it to bend and this deflection is used to construct the image (see Fig. 2). The 
degree to which the probe tracks the surface depends both on the speed or gain of the feedback loop as well as the probe shape (tip radius, aspect ratio) as compared to the feature shape.

Both intermittent and non-contact modes are vibrating cantilever methods that rely on more subtle feedback mechanisms (see Fig. 3). Unlike contact mode, non-contact mode is performed in the attractive force regime. A stiff cantilever is used and is vibrated near its resonant frequency while the probe is kept at a distance of up to $10 \mathrm{~nm}$ above the surface. Changes in the resonant frequency are used as the feedback mechanism to adjust the distance between probe and the sample. In intermittent contact mode, the cantilever oscillates with sufficiently large amplitude that it experiences both the attractive and repulsive force with the surface, which causes it to lightly come into contact with the surface. The change in amplitude of the oscillation due to damping provides the feedback mechanism. Both non-contact and intermittent contact modes allow the interrogation of samples that otherwise could not be imaged.

A broad range of probes and feedback methods are available to image different aspects of surface interactions other than topography including magnetic, electric, thermal, chemical, and mechanical properties. Friction images are an example of one such mode (Fig. 2). As the probe scans over the surface it can interact with materials that have differences in either chemical or mechanical "stickiness" or friction. The cantilever then twists in response to this interaction and results in a lateral shift in position of the reflected laser beam into the detector that is used to build the image.

Deflection images are a convenient way to display information when the surface is too rough to visualize all the topographic information without the contrast becoming saturated. These images are constructed using only the vertical deflection of the cantilever (Fig. 2) and highlight changes in topography; in this sense the are akin to derivatives of the height image. This mode of imaging is particularly useful when imaging atomic step motion.

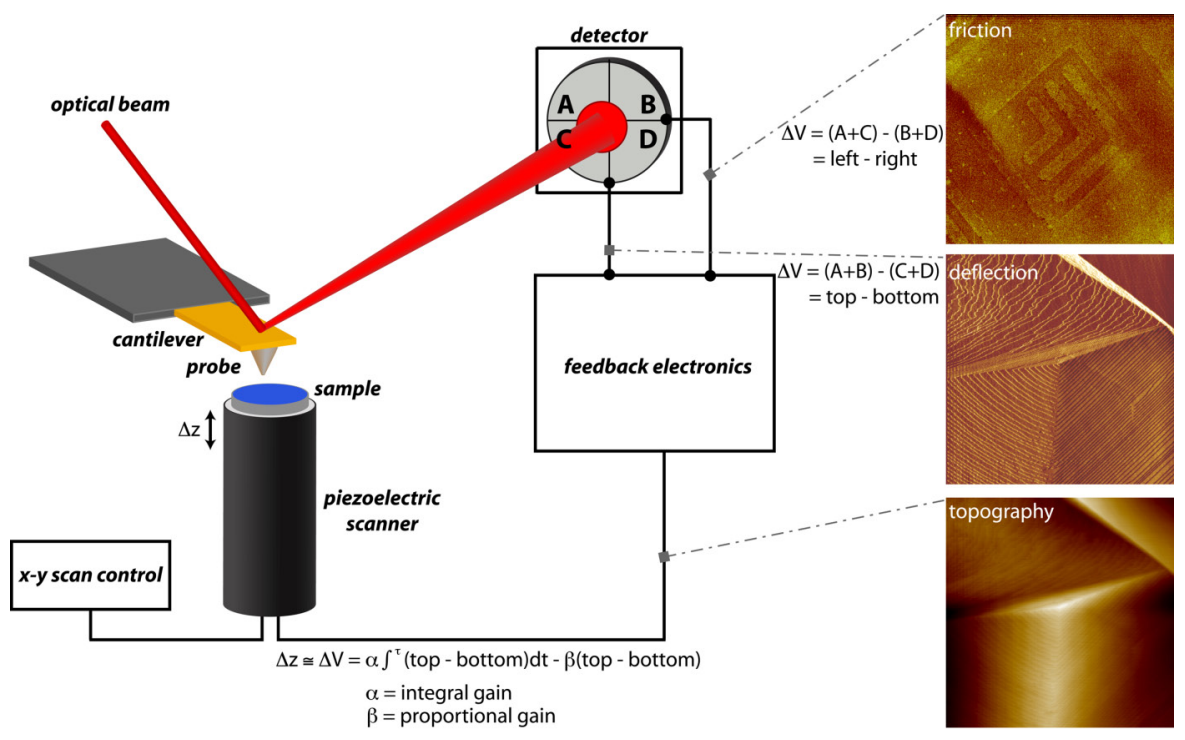

FIGURE 2. Schematic of the basic components of the scanning probe microscope along with a sample of the different types of images that one can acquire by sampling various detector signals. 


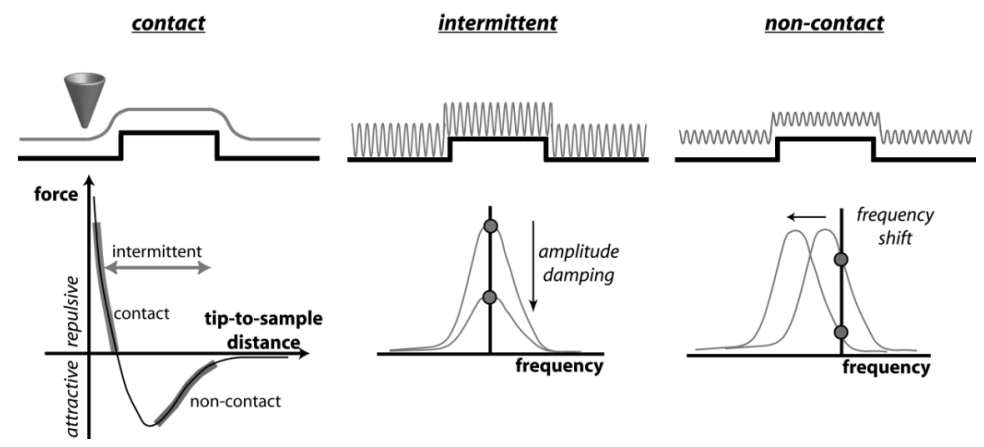

FIGURE 3. Schematic representation of the SPM modes used to acquire images and the forces that are probed in these modes. In contact mode, the tip probes the repulsive interaction. In intermittent mode the tip comes close enough to the surface that the oscillation becomes damped which both lowers the amplitude and broadening the frequency response (or quality factor, Q). By contrast, in non-contact mode, interactions with the surface shift the resonant frequency with less effect on the quality factor. In principle the frequency can shift to either lower or higher values depending on the interaction.

To image crystal growth in solution, the choice of operating mode is determined by how fast the surface is evolving and whether the tip force disturbs the surface. The scan rate must be sufficiently rapid to capture the surface events and the force must be sufficiently small so as not to distort the surface or influence the crystal growth. Contact mode has the most rapid image capture rate and for this reason is favored for monitoring dynamic step motion. However, for soft materials such as proteins it is sometimes necessary to use intermittent or non-contact modes to reduce the disruption of the surface.

One of the benefits of working fully immersed in solution is that capillary forces cannot accelerate the tip into the surface thereby degrading image resolution. Force curves, which are created by moving the cantilever vertically with respect to the substrate, illustrate this point (Fig. 4). As the tip approaches the surface, adsorbed water normally present under ambient conditions, forms a meniscus with the tip and draws it into to the surface. This force accelerates the tip towards the surface and causes a larger area of the tip apex to come into contact with the sample. As the contact area sets the image resolution, this typically degrades image quality. In fluid, capillary forces are eliminated and only the interaction forces are measured.

Any variation in the tip-sample distance due to thermal drifts or vibrations (typically acoustic or mechanical) will appear as noise on the image. For this reason the highest resolution instruments use a compact geometry and are made from materials that have low or similar thermal expansion coefficients. The fluid cell offers some protection from acoustic vibrations and one should feel free to sing (but not dance) while it is in use.

In summary, in situ AFM opens an exciting window into the study of solution crystal growth. For dynamics, typically images are collected in contact mode to take advantage of faster imaging conditions and deflection images are used to analyze step motion as these enunciate step-edges. Imaging and force measurement in fluid eliminates capillary forces and can improves image resolution. In addition, the fluid cell buffers some acoustic noise. New challenges present themselves in the form of leaks, bubbles and noise caused by solution flow, tubing and heating materials. These issues and some of their solutions will be discussed in later sections. 


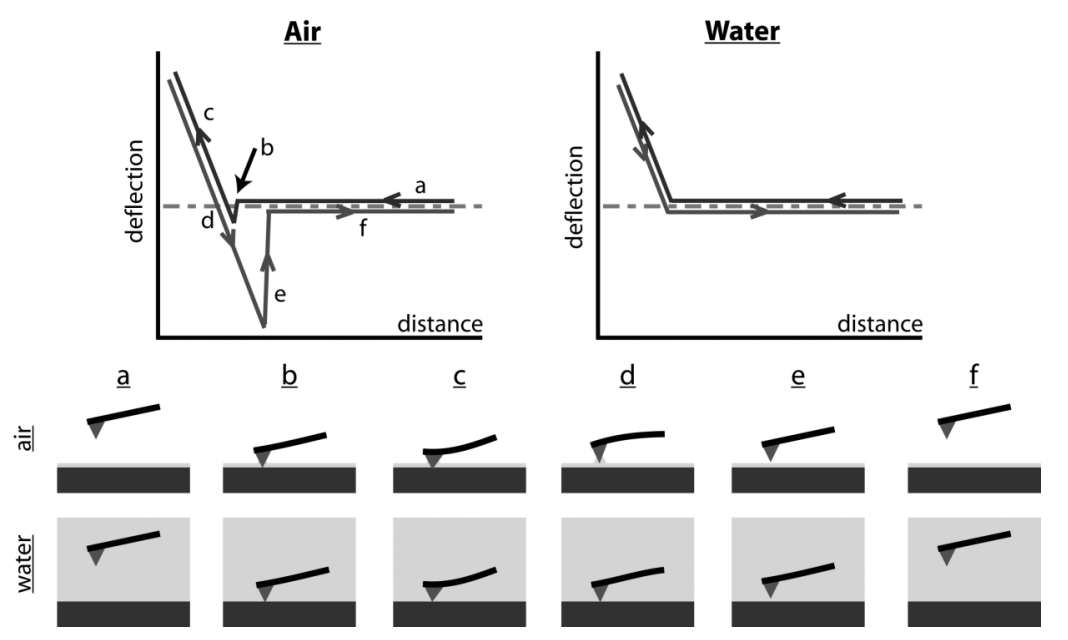

FIGURE 4. An illustration of the difference in force curves obtained in air and in fluid. In air there is a "snap to" contact (b-c) and a large "snap off" (d-e) that is caused capillarity forces between the probe and a thin layer of water on the surface. Performing force curved in fluid eliminates this complexity and directly measures the interaction forces between the probe and surface in that particular fluid. In this schematic the force between the surface and probe is neutral although, depending on the fluid, it can be either attractive or repulsive.

\section{SOLUTION SPECIATION}

Solution speciation is the starting point for developing a quantitative model of crystal growth and serves the essential role of translating crystal growth "recipes" into the parameters that drive crystal growth (Fig. 5).

Solution speciation techniques have been made routine with commercial and shareware programs. Such programs calculate the concentrations and activities of all solution ions and complexes from a list of initial reactants and a database of potential reactions with their respective association constants $\left(\mathrm{K}_{\mathrm{a}}\right)$ and solubility constants $\left(\mathrm{K}_{\mathrm{sp}}\right)$ [26]. Typically a solution is described in terms of the ion or salt concentrations used to prepare them however it is the activities rather than concentrations that determine the full speciation. Speciation shown in this chapter uses GeoChemists' WorkBench[27] with an extended Debye-Huckle formulae[28] to define the activity coefficients. Fig. 5 shows an example of the importance of solution modeling for a crystal growth experiment involving the calcium phosphate mineral brushite $\left(\mathrm{CaHPO}_{4} \cdot 2 \mathrm{H}_{2} \mathrm{O}\right)$. Starting with a solution recipe (left) one can then input the necessary $\mathrm{K}_{\mathrm{a}}$ 's and $\mathrm{K}_{\mathrm{sp}}$ 's into a database to calculate the activities of all species. The speciation diagram in the center shows how the activities of calcium and phosphate species change as a function of $\mathrm{pH}$. The fundamental growth units of brushite, $\mathrm{Ca}^{2+}$ (blue) and $\mathrm{HPO}_{4}{ }^{2-}$ (red), are displayed using solid lines while the other phosphate and calcium-phosphate complexes are displayed using broken lines $(\mathrm{Na}, \mathrm{K}$, and $\mathrm{Cl}$ species are included in the calculation but left off the graph for clarity). Then by specifying the $\mathrm{pH}$ and $\mathrm{T}$ one can calculate the other crystal growth parameters (right). These parameters will be discussed at length in the next section. 


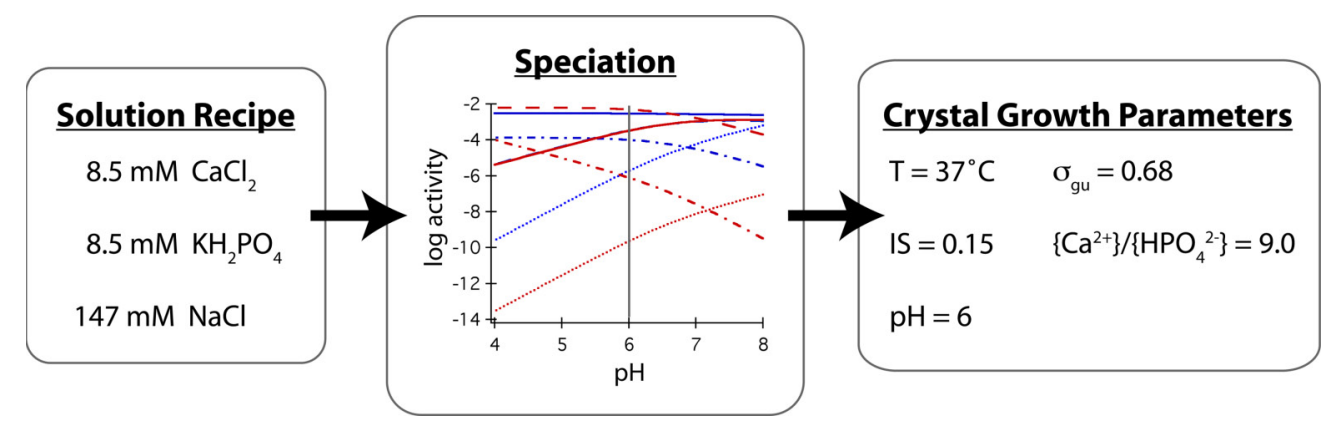

FIGURE 5. An example of how solution speciation can be used to specify the important growth parameters for crystal system using brushite $\left(\mathrm{CaHPO}_{4} \cdot 2 \mathrm{H}_{2} \mathrm{O}\right)$. The activities of the following ions are plotted on the diagram in the center. BLUE: $\mathrm{Ca}^{2+}$ (solid), $\mathrm{CaPO}_{4}{ }^{-}$(dotted), $\mathrm{CaHPO}_{4(\mathrm{aq})}$ (dashed), $\mathrm{CaH}_{2} \mathrm{PO}_{4}^{+}$ (dot-dash). RED: $\mathrm{HPO}_{4}{ }^{2-}$ (solid), $\mathrm{PO}_{4}{ }^{3-}$ (dotted), $\mathrm{H}_{2} \mathrm{PO}_{4}{ }^{-}$(dashed), $\mathrm{H}_{3} \mathrm{PO}_{4(\mathrm{aq})}$ (dot-dash). Consult the text for additional details.

\section{CRYSTAL GROWTH PARAMETERS}

This section will briefly discuss how crystal growth or dissolution is affected by the composition of the solution, including the effects of supersaturation, $\mathrm{pH}$, ionic strength and ratio of cations to anions. In general these parameters can affect both the solution speciation as well as the surface of the mineral. The effects of each of these parameters are summarized in Table 1.

TABLE 1. Crystal Growth Parameters.

\begin{tabular}{|c|c|c|}
\hline Parameter (Symbol) & Effect on Bulk Solution & Effect on Surface \\
\hline Supersaturation $(\sigma)$ & Stability of solid phases & $\begin{array}{l}\text { Net flux to surface; determines } \\
\text { growth mode (island vs. step) }\end{array}$ \\
\hline $\mathrm{pH}$ & $\begin{array}{c}\text { Solution speciation (and } \\
\text { subsequently supersaturation) }\end{array}$ & Net charge of surface; protonation \\
\hline Ionic Strength (I) & $\begin{array}{l}\text { Screening length within the } \\
\text { solution - activity coefficients }\end{array}$ & Debye length of the double layer \\
\hline Temperature $(\mathrm{T})$ & $\begin{array}{l}\text { Solution speciation through } \\
\text { temperature dependence of } \\
\text { association constants }\end{array}$ & $\begin{array}{l}\text { Kinetics of adsorption, desorption, } \\
\text { diffusion }\end{array}$ \\
\hline Ionic ratio of growth units & Solution speciation & $\begin{array}{l}\text { Kinetics of incorporation; activation } \\
\text { barriers for different ions }\end{array}$ \\
\hline Additive concentration ([X]) & $\begin{array}{c}\text { Solution speciation (and } \\
\text { subsequently supersaturation) }\end{array}$ & $\begin{array}{l}\text { Various: step-pinning, surfactant } \\
\text { blocking layer, incorporation, etc. }\end{array}$ \\
\hline
\end{tabular}

The most important crystallization parameter is the thermodynamic driving force or the supersaturation. The supersaturation, $\sigma=\Delta \mu / k T$, is a unitless number proportional to the chemical potential difference associated with molecules transferring from the bulk solution to the bulk solid phase and can be determined from speciation calculations. Three related representations for the driving force are found in the literature: the supersaturation, the supersaturation ratio, and the relative supersaturation. It is instructive to spend a moment to define the relationship between these terms.

The supersaturation ratio can be computed by using the solution speciation results to calculate the ion activity products $(I P)$ for minerals of interest. The supersaturation ratio, $S$ is then given by 


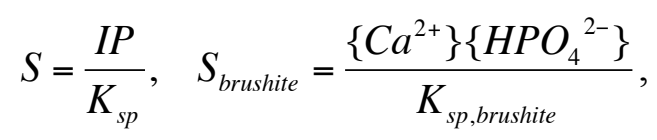

where the supersaturation ratio for the calcium phosphate mineral brushite, is shown explicitly. For $S=1$, the mineral and solution are in equilibrium, for $S<1$ the solution is undersaturated and the mineral will dissolve, and for $\mathrm{S}>1$ the solution is supersaturated and the mineral will grow. In this example brushite has two growth units. Traditionally the supersaturation is written per molecule rather than per growth unit and is related to $\mathrm{S}$ through $\sigma=\Delta \mu / k T=\ln S$. To allow comparisons between materials with different numbers of growth units, it is useful to define a driving force that is normalized for the number of growth units in the unit cell $(n)$,

$$
\sigma_{g u=} \frac{\Delta \mu_{g u}}{k T}=\ln S^{\frac{1}{n}}
$$

where 'gu' denotes growth unit. Notice that for $\mathrm{S} \sim 1$ (i.e. near saturation) the logarithm can be expanded ( $\ln x \sim 1-\mathrm{x}$ for $\mathrm{x} \sim 1$ ) leading to what is commonly termed, the relative supersaturation, defined as:

$$
\sigma_{r e l}=S^{1 / n}-1 \quad .
$$

For $\sigma, \sigma_{g u}$, and $\sigma_{\text {rel }}$ positive values imply crystal growth. However most solutions have a metastable region, where the solution is supersaturated but not enough to overcome the energy barrier that prevents crystals from spontaneously precipitating from solution phase (on reasonable timescales). In this region, crystal growth occurs on existing crystal surfaces without nucleating new crystals. Above a supersaturation threshold the solution becomes unstable and both nucleation and growth occur; here crystals are said to "crash" out of solution.

Fig. 6 demonstrates the importance of supersaturation in crystal growth. As the supersaturation ratio increases both the kinetics and the hillock geometry change, going from slow step velocities and widely spaced steps at low supersaturation to high step velocities and densely spaced steps at high S. The details of these kinetic and morphological affects of supersaturation will be discussed later in this chapter.

The second parameter, $p H$ affects both the solution as well as the mineral surface. In mineral systems a shift to lower $\mathrm{pH}$ will lower the saturation state. For example, in phosphate systems this occurs by shifting the balance of phosphate species from $\mathrm{PO}_{4}{ }^{3-}$ to $\mathrm{HPO}_{4}{ }^{2-}$ to $\mathrm{H}_{2} \mathrm{PO}_{4}$ as the $\mathrm{pH}$ is lowered and similarly in carbonate systems lower $\mathrm{pH}$ shifts species from of $\mathrm{CO}_{3}{ }^{2-}$ to $\mathrm{HCO}_{3}{ }^{-}$to $\mathrm{H}_{2} \mathrm{CO}_{3}$. At the mineral surface the $\mathrm{pH}$ can shift the surface charge by changing the distribution of proton and hydroxyl groups hydrating the interface. In principle these may change the activation barriers associated with crystallization.

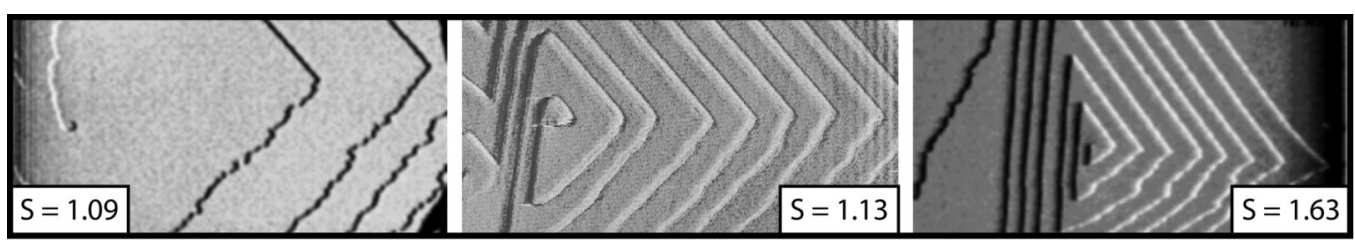

FIGURE 6. Images of atomic steps emerging from a dislocation on the (010) surface of brushite, $\mathrm{CaHPO}_{4} \cdot 2 \mathrm{H}_{2} \mathrm{O}$, during an in situ SPM crystal growth experiment. This series of images demonstrates that the step spacing increases due to a decrease in the critical length with increasing supersaturation. All images are $6 \mu \mathrm{m} \times 3 \mu \mathrm{m}$. 
The third parameter, ionic strength plays a role in screening both ion-ion electrostatic interactions in solution (which is accounted for by the activity coefficient) and electrostatic interactions between ions in solution and the surface. The ionic strength of a solution, $I$, is defined as

$$
I=1 / 2 \sum_{i}[i] z_{i}^{2}
$$

where $[i]$ is the concentration and the $z_{i}$ the charge, of each ionic species, $i$. The Debye length sets the screening range at the mineral surface and thus sets a length scale for the electric field generated at the charged surface. At distance greater than the Debye length, the electric field is effectively shielded and therefore does not affect charged species. An ionic strength near $0.15 \mathrm{M}$ corresponds to a Debye length of approximately $1 \mathrm{~nm}$.

Temperature is an important crystal growth parameter changing both the thermodynamic and kinetic aspect of crystal growth. Solution speciation changes with temperature because solubility products and association constant are temperature dependent. Temperature also effects the kinetics of adsorption, desorption, and diffusion. Within transition state theory, these motions are typically modeled as activated hopping processes where the probability of making the jump can be written as $P=v(T) e^{-E_{a} / k T}$. The attempt frequency, $v$, is weakly temperature dependent and is typically treated as independent of temperature. Thus, the primary temperature dependence is the exponent.

The cation to anion activity ratio (for example of calcium to phosphate or calcium to carbonate) acknowledges that growth rates may not be only dictated by the supersaturation and the surface energies but rather that kinetics may play a role. In principle ion ratios can affect growth rates, growth shape, and the transformation of metastable phases. The growth of a multi-species crystal relies on the relative rates of adsorption and desorption of the various ions or growth units that make up the unit cell. For a simple salt such as $\mathrm{NaCl}$ the growth units are the $\mathrm{Na}^{+}$and $\mathrm{Cl}^{-}$ions. However in general the growth units represent the pathway with the lowest activation barrier that allows an ion to move from the solution state to the solid state and vice-versa. In a binary ionic compound such as brushite $\left(\mathrm{CaHPO}_{4} \cdot 2 \mathrm{H}_{2} \mathrm{O}\right)$, it is tempting to think of the growth units as $\mathrm{Ca}^{2+}$ and $\mathrm{HPO}_{4}{ }^{2-}$, but it is possible that in the process of shedding waters of hydration and incorporating into the solid that the activation barrier is lower for a multistep process wherein one of the other phosphate complexes (e.g. $\mathrm{H}_{2} \mathrm{PO}^{4-}$ or $\mathrm{PO}_{3}{ }^{3-}$ ) adsorbs and then adds or sheds a hydrogen. Many groups have shown that ion ratios play a role in kinetics and some progress has been made at modeling these effects[5]; however, most crystal growth models assume a single species and more work is needed to fully describe multi-component crystals.

\section{ENVIRONMENTAL CONTROL}

The control of environmental conditions is an essential component of quantitative crystal growth. Most crystal growth experiments require auxiliary controls beyond the operation of the AFM; these include flow control, temperature control, and, for electrochemical deposition, electrochemical control. It is important when attaching auxiliary controls to the fluid cell that these not couple vibrations into the microscope. For this reason, floppy materials, (probes, wires, or tubing that have small spring 
constants and that damp vibrations) should be used when connecting pumps or electronics to the fluid cell.

Flow control is achieved by using a pump or gravity fed system. A typical setup is shown in Fig. 7 where a peristaltic pump is used. This type of pump is convenient when the solution must be continuously monitored for $\mathrm{pH}$ or temperature or when the experiment requires that the solution be easily exchanged or modified by additives. The addition of a vibration damper prevents the transmission of pulses from the pump to the fluid cell, which otherwise would degrade imaging. A syringe pump has no mechanical pulsing and may be used in cases where the solution does not need to be continuously monitored or modified.

For quantitative analysis it is usually necessary to have a well-defined supersaturation. For this reason the flow rate must be sufficiently high to ensure that crystal growth is not limited by mass transport of ions through the solution to the surface. In practice this is achieved by increasing the flow rate until step speed is independent of flow rate. However, recent finite element simulations[29] of flow dynamics in an SPM fluid cell have shown that solute is depleted in areas surrounding growing calcium oxalate monohydrate $(\mathrm{COM})$ crystals caused by the inability of the conductive mass transport to replenish solute in these areas. For COM crystals directly under the cantilever (i.e. those being imaged) this depletion is unaffected by flow rate and results in lowering the relative supersaturation from the inlet value by $\sim 3 \%$. Although this supersaturation change may be small for COM it might be more significant for crystals composed of slow diffusing or large molecules or those with faster growth rates. Therefore, fluid dynamics should be considered when making kinetic measurements of these systems.

As stated earlier, temperature affects both the thermodynamics and kinetics of crystal growth. For experiments where the solution must be monitored for $\mathrm{pH}$ or other ions, the temperature can be controlled external to the fluid cell by either passing the fluid through a peltier device or by keeping the solution reservoir in a thermostated box. The tubing between the heat source and the fluid cell should be short to minimize temperature drop or, alternatively, insulated tubing can be used. It is also advisable to use small thermocouples that can be threaded up the out-port to directly monitor the temperature in the fluid cell. These external methods work well for experiments that are carried out under flow conditions ( $>1 \mathrm{~mL} / \mathrm{min}$ ) as the flow rate will affect the temperature inside the fluid cell. For slow flow or no flow experiments, the temperature can be controlled by placing the sample directly on a peltier device (low flow) or in a copper cup that is in contact with a peltier device (no flow).

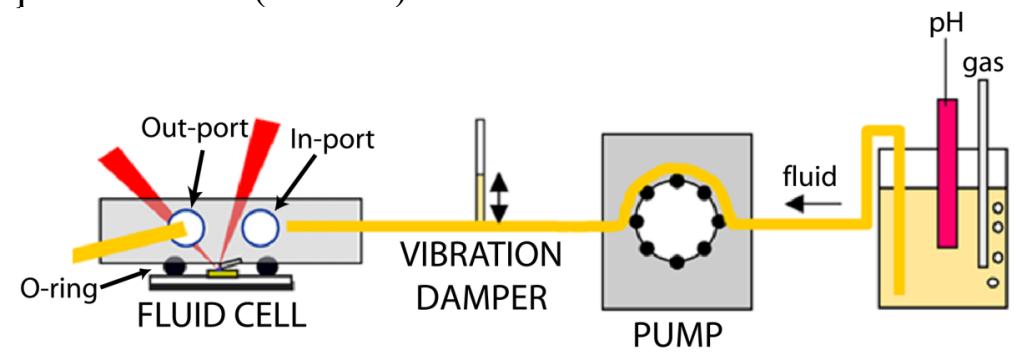

FIGURE 7. A schematic drawing that shows a common fluid cell setup where a peristaltic pump is used. This particular fluid cell is made of glass and uses a silicone o-ring. In this configuration the distance between the bottom of the fluid cell and the sample and the o-ring diameter define the internal volume which in this case is $\sim 50 \mu \mathrm{L}$ 


\section{MONITORING CRYSTAL GROWTH}

This section will describe the types of in situ crystal growth measurements that can be made using SPM. These measurements include the velocity of atomic steps; the critical length for step motion and the critical island size; the morphology of steps and islands; the fluctuations of step edges. What these measurables tell you about crystal growth are summarized in Table 2. We will focus our discussion on step growth rather than island growth.

TABLE 2. A summary of measurable quantities common in SPM experiments

\begin{tabular}{cc}
\hline Step Measurable & Determines \\
\hline Step velocity & Kinetic coefficient $(\beta)$ \\
Critical step length & Step edge free energy $(\gamma)$ \\
Morphology & Pinning; New step/facet expression \\
Step edge fluctuations & Kink attachment/ detachment rates; Kink density \\
\hline
\end{tabular}

\section{Measuring step velocity}

The measurement of atomic steps spaced $>\sim 10 \mathrm{~nm}$ and moving with rates in the range of $1-100 \mathrm{~nm} / \mathrm{s}$, plays to the strengths of commercial scanning probe microscopes (that is, atomic resolution in the z-direction, tip diameters $\sim 10 \mathrm{~nm}$, and scan rates up to $\sim 15 \mathrm{~Hz}$ without extreme image degradation). For this reason the bulk of our discussion will focus on step velocity measurements. The step velocity, in the simplest case of an infinitely long step with high kink density, is given by:

$$
v=\beta \Omega\left(I P-K_{s p}\right)=\beta \Omega K_{s p}(S-1),
$$

where $I P, K_{s p}$ and $S$ are the ion activity product, solubility product, and supersaturation ratio respectively (as defined in Equation 1 but in units of number of atoms per volume instead of the more traditional moles per liter), $\Omega$ is the atomic volume, and $\beta$ is the kinetic coefficient with units of velocity. There are many exceptions to this form including variations as a function of step length[30,31], variation due to low kink density[20,21], variation due to multiple kink types[32], and variation due to impurity interactions. (This last topic is covered in the chapter by J. J. De Yoreo within this volume and will not be discussed here.) Nevertheless, where it holds one can see that plots of the step velocity as a function of the supersaturation ratio serve to define the kinetic coefficient, an important quantity that contains (and hides) all of the physics associated with the desolvation, adsorption, diffusion, and incorporation.

Measuring step motion is straightforward although complicated by the fact that in a scanned image each pixel is acquired at a different time. Thus, dynamic measurements require the decoupling of the scanning motion from the feature motion. We will discuss three ways to measure the velocity of steps or other features moving on surfaces: (1) measurement with respect to a fixed object; (2) measurement in disabled mode; and (3) measurement using apparent step angle. In all cases typically one tries to find the site of a screw dislocation because at this position, steps are continuously sourced and all step directions can be imaged simultaneously.

The first and most intuitive method is the displacement of a step with respect to a fixed position on the surface. The fixed point could be a natural defect, such as the center of a growth hillock, or a man-made fiducial mark such as a nanoindentation or a pattern of a 
different material. Velocity measurement using a fixed feature are simplified by using subsequent images captured under identical imaging conditions (such as scan direction, scan rate, scan angle etc.) An example of this method is shown both schematically and with an example in Fig. 8a using the dislocation source as the fixed point. As shown, a line is drawn from the fixed point to the step edge in a direction perpendicular to the step. The intersection of the perpendicular line and the step edge defines a point with both spatial coordinates as well as a time $\left(x_{1}, y_{1}, t_{1}\right)$. A similar procedure occurs for the same step in a later image, defining $x_{2}, y_{2}$, and $t_{2}$. As a quick check to ensure that the same step is being measured, the steps can be extrapolated to the same time-point at the edges of the image. In the example shown, which is imaged in the scan-down direction, the step position extrapolated to the bottom of the first image matches the step position extrapolated to the top of the second image because these points represent approximately the same time. The velocity is obtained using pixel arithmetic. The time at a pixel $\left(x_{i}, y_{i}\right)$ is given by $t_{i}=T_{i}+\left[x_{\text {pix }} y_{i}+x_{i}\right] / v_{\text {tip }}$ where $T_{i}$ represents the start time of the image, $x_{\text {pix }}$ is the number of pixels per line, and $v_{t i p}$ is the velocity of the tip in pixels per second given by $v_{t i p}=2$ (scan rate) (scan size). The elapsed time $(\Delta t)$ and displacement $(\Delta d)$ between the measurements is then:

$$
\begin{gathered}
\Delta t=t_{2}-t_{1}=\left(T_{2}-T_{1}\right)+\frac{\left[x_{p i x}\left(y_{2}-y_{1}\right)+\left(x_{2}-x_{1}\right)\right]}{v_{t i p}} \\
\Delta d=a\left(\frac{n m}{p i x}\right) \sqrt{\left(x_{2}-x_{1}\right)^{2}+\left(y_{2}-y_{1}\right)^{2}},
\end{gathered}
$$

where $a(\mathrm{~nm} / \mathrm{pix})$ is the conversion factor from pixels to spatial dimensions.

The second method involves disabling the slow scan (vertical) axis so that the tip only rasters over a single scan line. The image that is constructed displays distance (horizontal axis) vs. time (vertical axis). The most straightforward case is presented in Fig. 8b where the steps have been oriented perpendicular to the fast scan direction so that the vertical axis is not a convolution of both distance and time. In this case, two well-spaced points are chosen along the slope and the elapsed time is calculated as above but the displacement is simply $\Delta d=x_{2}-x_{1}$. Of course, measurements can still be made when the step is at a random angle with respect to the fast scan direction. In this case the true step angle must be calculated by comparing the apparent step angle between up and down scans as shown in Fig. 8c. The process for finding the true angle is discussed below.

In the third method, the change in angle between the two scan directions gives a measure of both the true step angle and the step velocity as given by the equations:

$$
\begin{aligned}
& \tan \varphi_{0}=\frac{4 x_{p i x} m_{u} m_{d}+m_{u}+m_{d}}{2\left[x_{p i x}\left(m_{u}+m_{d}\right)+1\right]} \\
& \frac{v}{v_{t i p}}=\frac{m \cos \left(\varphi_{0}\right)-\sin \left(\varphi_{0}\right)}{2 x_{p i x} m+1}
\end{aligned}
$$

where $m_{i}$ is the apparent slope of the step in up $\left(m_{u}\right)$ and down $\left(m_{d}\right)$ scanned images, $\tan \left(\varphi_{0}\right)=m$ is the true slope of the step, $v$ is the true velocity of the step, and $x_{\text {pix }}$ and $v_{\text {tip }}$ are as defined above. 


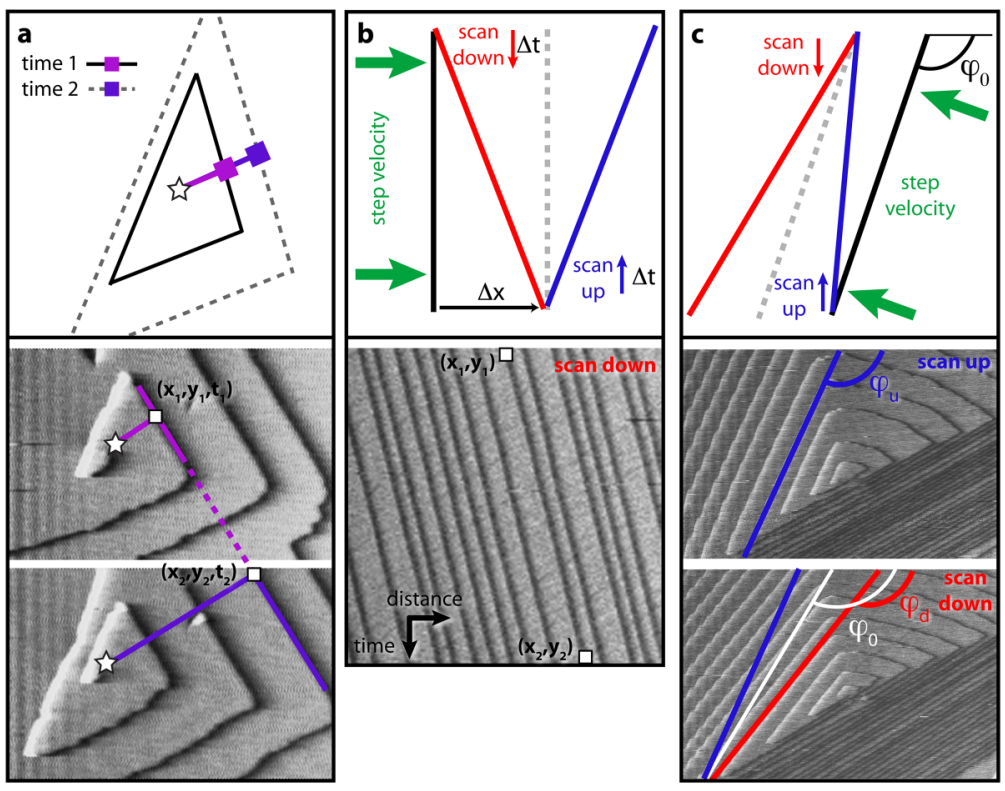

FIGURE 8. Schematic illustrations and in situ AFM images of brushite growth that show the three types of step velocity measurements: a) measurement of step displacement and elapsed time from a dislocation source, b) measurement of step displacement and time from a disabled scan, and c) measurement of step velocity from the apparent step angle in up and down scanned images.

Step velocities provide a wealth of information related to the activation barriers that set the rate at which ions or molecules transfer between the solution and the solid phase. The major difficulty with interpreting velocity measurements is that they are sensitive to most solution parameters. For example, one expects the step velocity to vary with $\mathrm{pH}$, supersaturation, temperature, impurity concentrations, and, in multicomponent systems, the ratios of species. Thus, interpretation relies on detailed knowledge of the solution state, motivating why much of this chapter is devoted to defining solution parameters.

Quantitative measurements of velocity versus supersaturation are found in the literature for a variety of solution grown crystals including minerals such as calcite[33, 34], barite[35, 36], hydroxyapatite[37]; optical crystals such as ammonium dihydrogen phosphate (ADP)[38] and potassium dihydrogen phosphate (KDP)[39]; several proteins[40-44]; and organic crystals such as hydrogen bonded tapes[45] and uric acid[46]. Land and De Yoreo tabulate kinetic coefficients for several systems[41] demonstrating that they vary over several orders of magnitude. The study of velocity versus other solution variables such as ionic strength[47], $\mathrm{pH}[43,46]$, and temperature [36, 38, 48] have also been quantified.

Note that variables such as $\mathrm{pH}$, temperature, ionic strength, and ion ratios, typically also affect the supersaturation and must be properly accounted for in the speciation to decouple the two effects. This is nicely illustrated in a study of brushite $\left(\mathrm{CaHPO}_{4} \cdot 2 \mathrm{H}_{2} 0\right)$ crystal growth where variations in species ratios were used to determine which ion limited growth. For brushite, the supersaturation ratio can be increased either by increasing the $\mathrm{Ca}^{2+}$ ion concentration or the $\mathrm{HPO}_{4}{ }^{2-}$ ion concentration. Plots of the step velocity versus S-1 (Fig. 9) shows that the kinetic coefficients (slope of the line) change under these two conditions. This implies that the activation barriers under conditions of excess $\mathrm{Ca}^{2+}$ and excess $\mathrm{HPO}_{4}{ }^{2-}$ differ. This is illustrated more directly by plotting the 
normalized velocity as a function of the ratio of $\mathrm{Ca}^{2+}: \mathrm{HPO}_{4}{ }^{2-}$ under conditions where the supersaturation has been held constant. The fast rates with excess $\mathrm{HPO}_{4}{ }^{2-}$ and low rates with excess $\mathrm{Ca}^{2+}$ suggest that $\mathrm{HPO}_{4}{ }^{2-}$ incorporation is the rate-limiting step for these crystals.

The velocity also varies as a function of step length for lengths near the critical length. Below the critical length, the step does not propagate and thus has a velocity of zero, whereas the infinitely long step has the form suggested in Eq. 4. But how does it vary between these two extremes?
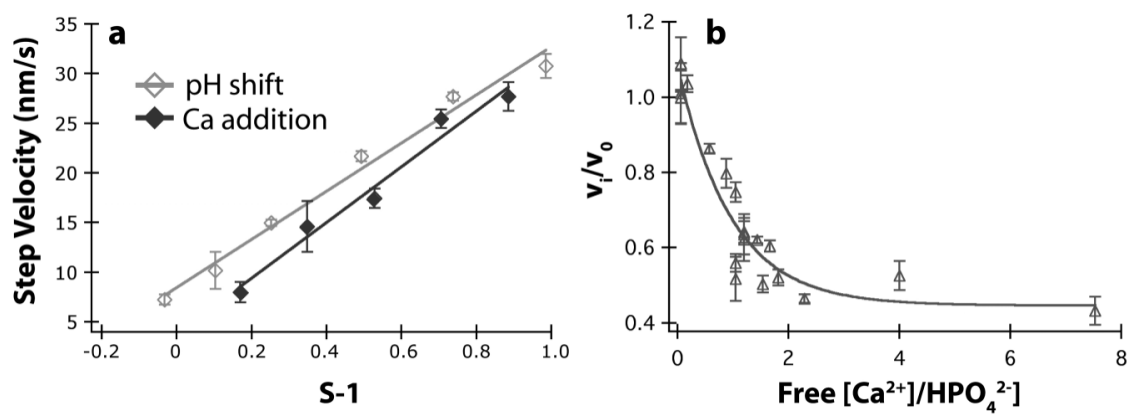

FIGURE 9. Plots illustrating the types of velocity measurements that can be made. a) A plot of step velocity versus supersaturation ratio for two types of crystal growth experiments on brushite. b) A plot demonstrating that step velocities change with ratio of ionic species when the supersaturation is held constant. This data demonstrates that the kinetic coefficient is highly sensitive to ion ratios.

\section{Measuring the critical length or the critical island size}

For steps emanating from dislocation hillocks, SPM can be used to directly measure the critical step length that results from the Gibbs-Thomson relation[49-51]. As a step grows, molecules move from the solution phase to the solid phase, reducing the energy of the system however, this creates more step-edge, which increases the energy of the system. The critical length is the length at which these two opposite influences balance. When a row of molecules is added to an existing step (Fig. 10a), the change in Gibbs free energy is:

$$
\Delta G=-n \Delta \mu+2 a_{1} h \gamma
$$

where $n$ is the number of molecules that add to the step, $\Delta \mu$ is the change in chemical potential per molecule, $a_{l}$ is the dimension of one molecule in the direction of the new edge, $h$ is the height of the step, and $\gamma$ is the step-edge free energy in units of energy per area. Note that the length parallel to the step does not count as new edge as it existed before the row of molecules was added. The step length is related to the number of molecules by $L=n / a_{2}$ where $a_{2}$ is the dimension of a molecule parallel to the step. Setting $\Delta G$ to zero and solving for the length one obtains:

$$
L_{c}=\frac{2 a_{1} a_{2} h \gamma}{\Delta \mu}=\frac{2 \Omega \gamma}{k T \ln S}
$$

In general, the critical length will vary depending on the step, reflecting the orientation dependent step-free energy. In a given image (Fig. 10b) one can determine whether the step emerging from the dislocation source is stationary (sub-critical) or moving (supercritical) by comparing its location relative to the dislocation source. After measuring the 
length of this initial step over numerous sequential images, the critical length is bounded by determining the longest sub-critical step and the shortest supercritical step.

The critical step length for a dislocation hillock was measured directly for the first time using this method[30] demonstrating the orientation dependence and the scaling with supersaturation (Fig. 10b). A similar method can be used to estimate the critical island size for two-dimensional nucleation[52]. Unlike step growth from dislocation sources, sub-critical islands shrink rather than remain stationary, while supercritical islands grow. Finding the maximum size for shrinking islands and the minimum size of growth islands gives a range for the critical island size.

It should be noted that the Gibbs-Thomson relation implicitly assumes that steps are populated with kinks and that kink-nucleation is not a rate-limiting step for propagation. There is gathering evidence[21, 32] suggesting that in some systems this may not hold. In this scenario, step propagation is kinetically limited due to the low probability of nucleating a kink, which is a necessary precursor to the facile addition of new molecules. The critical length then reflects the probability of nucleating kinks rather than the stepfree energy. This discrepancy suggests that it will become important to couple critical length measurements with independent surface energy measurements and to define a theory that can accommodate kink-nucleation dynamics.
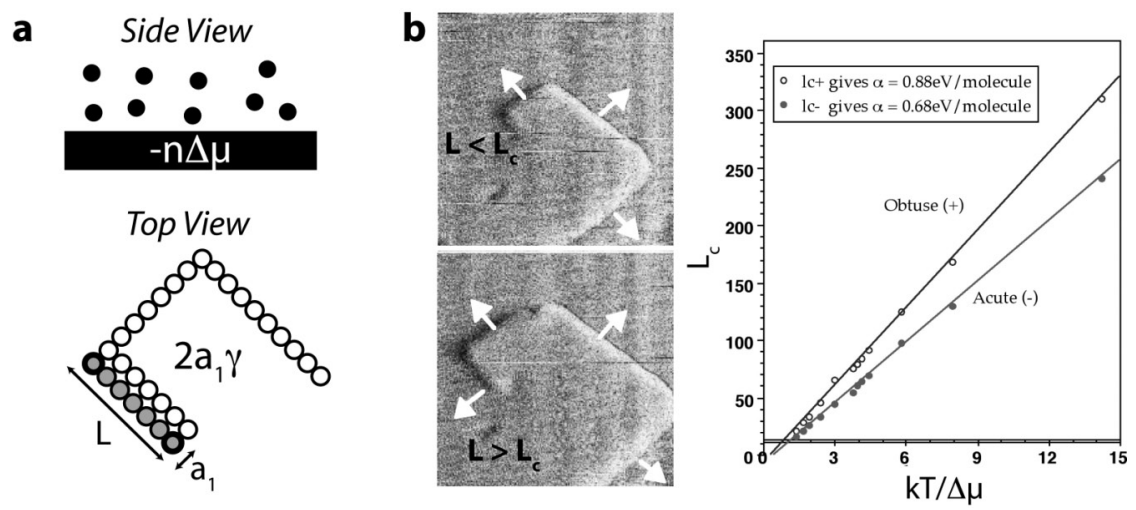

FIGURE 10. Examples of calcite growth showing the importance of the critical length in crystal growth. Sequential AFM images illustrate the measurement of $\mathrm{L}_{\mathrm{c}}$. In the first image, three of the four steps are propagating while the fourth is not because its length is less than the critical length. In the second image, the length of this step has surpassed its critical length and is propagating perpendicular to its edge. For calcite the critical lengths were used to determine the step edge free energy of the acute and obtuse steps. After[30]

\section{Geometric effects on kinetics}

Geometry plays a role in step kinetics. We will discuss two cases: the first we have already alluded to which is the effect of step length on velocity and the second, is the effect of merging hillocks that can act as sources of kinks.

The step velocity clearly depends on the length of the step as we have already shown that steps do not move for step lengths smaller than the critical length and that their velocity rises to a value represented by Equation 4, for long steps. More generally,

$$
v(L)=\beta \Omega K_{s p} S\left(1-S^{\left(\frac{L_{c}}{L}-1\right)}\right)
$$


which reduces to Equation 4, in the limit $L_{c} / L<<1$. Closer examination of this relationship in calcite growth (Fig. 11a) has shown that the velocity rises from zero to its infinite length value faster than is predicted by this equation and is one piece of evidence suggesting that the assumption of high kink density may not be valid for sparingly soluble minerals with straight steps[cite Teng].

There is also evidence that step kinetics depend on the presence of inside corners. Consider two singular steps that have different crystallographic orientations and propagate to meet one another. When they meet, they form an inside angle where there is no potential barrier for kink generation. Therefore, this angle can serve as a kink source that can accelerate step propagation when motion is limited by kink-nucleation. Indeed as Fig 11b shows, on brushite $\left(\mathrm{CaHPO}_{4} \cdot 2 \mathrm{H}_{2} \mathrm{O}\right)$, when the steps [201] and [101] touch one another, the [201] step is accelerated. This step acceleration implies that there is a higher kink generation rate due to the presence of the re-entrant corner.
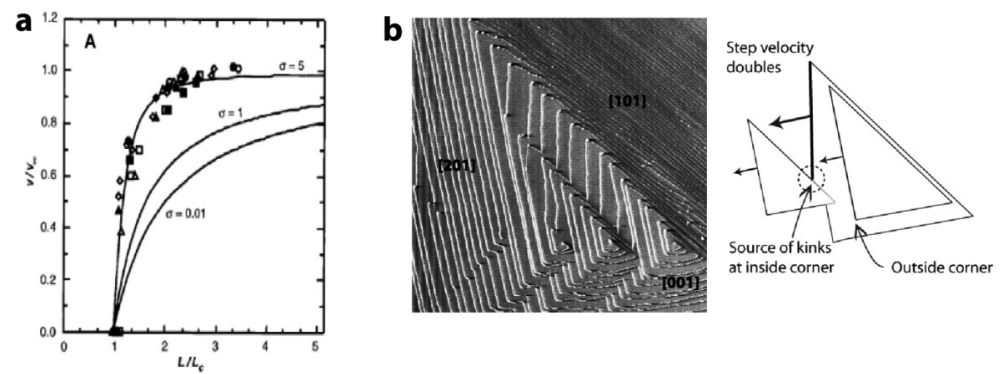

FIGURE 11. Examples demonstrating geometric effects on the step kinetics. a) Normalized velocity as a function of step length normalized to the critical step length. The velocity rises to its infinite step value more quickly than is predicted by Eq. 7 (After [30]). b) Atomic force microscope image $(7 \mu \mathrm{mx} 7 \mu \mathrm{m})$ of multiple merging hillocks on a growing brushite $\left(\mathrm{CaHPO}_{4} \cdot 2 \mathrm{H}_{2} \mathrm{O}\right)$ surface. The velocity of the [201] step direction (noted with arrows in the schematic) depends upon the corner type and roughly doubles when it terminates at an inside rather than an outside corner. In scanned images the observed step angles are a function of both the crystallographic orientation and the step velocity. To ascertain that the velocity doubled rather than the angle changed, images were compared scanning both down and up.

\section{Hillock geometry (step density)}

The critical length $\left(L_{c i}\right)$ and the step velocity $\left(v_{i}\right)$ for step direction, $i$, both affect the step density (or terrace widths, $w_{i}$ ) near the apex of a hillock. The terrace width in a particular direction depends on the time for a full revolution of the spiral $(T)$ and the velocity of the step, $w_{i}=v_{i} T$. Thus, the relative terrace widths reflect the relative velocities, $w_{1}: w_{2}: w_{3} \Leftrightarrow v_{1}: v_{2}: v_{3}$, and once the velocity is known in one orientation the others can be determined from the step densities. The time for a spiral revolution is a function of the velocities and the critical lengths in all directions. If one makes the simplifying assumption that the velocities are 0 for lengths less than $L_{c}$ and $v_{\text {inf }}$ for all other lengths, then for a triangular hillock the time is given by the sum of the times needed to grow each critical length:

$$
T=\left|\frac{L_{c 1} \operatorname{Sin} \theta_{1}}{v_{2}}\right|+\left|\frac{L_{c 2} \operatorname{Sin} \theta_{2}}{v_{3}}\right|+\left|\frac{L_{c 3} \operatorname{Sin} \theta_{3}}{v_{1}}\right|
$$


Figure 12 illustrates the effect of changing the critical length and the velocity on hillock geometry. Starting from a hillock with isotropic velocities and critical lengths, the first column demonstrates the effect of changing only one direction whereas the second column demonstrates the effect of changing all directions simultaneously. In particular changes to the critical length (whether isotropic or anisotropic) change the overall density of steps whereas only anisotropic changes to the step velocity affect the step densities.

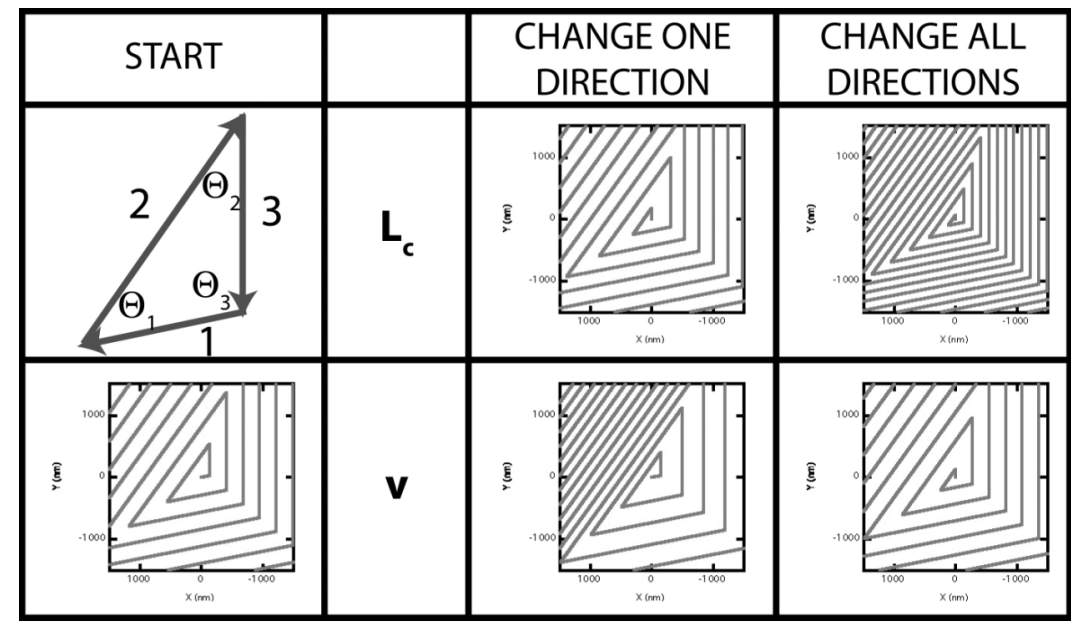

FIGURE 12. Example of the effects of isotropic and anisotropic changes in critical length $\left(\mathrm{L}_{\mathrm{c}}\right)$ and step velocity (v) on hillock geometry. When $\mathrm{L}_{\mathrm{c}}$ is changed the spiral period is affected changing the densities of all step-directions. Only anisotropic changes in the velocity change the step densities.

These effects explain the change in hillock geometry caused by increasing the supersaturation (Fig. 6). When the supersaturation is increased the velocity of all of the steps increases in approximately the same way thus while the steps move faster the hillock geometry does not change (Fig. 12, bottom row). However, the critical length also changes with supersaturation leading to a higher density of steps.

\section{OUTLOOK}

While most of the examples shown in this chapter are minerals evolving in dilute aqueous solutions, the SPM is an extremely flexible technique that can readily adapted to more challenging environments. To illustrate this point we show four examples in (1) aggressive organic solvents (Fig13a), (2) at low temperatures (Fig13b), (3) in corrosive environments (Fig13c), and (4) under electrochemical control (Fig13d). In the first example a 1,3,5-triamino-2,4,6-trinitrobenzene (TATB) crystal is shown dissolving and growing in dimethyl sulfoxide-based solvents[53]. Atomic step motion can be imaged although the solvent has an order of magnitude higher viscosity than water and is fluorescent. Imaging of the steps is being used to bound the solubility. In the second example, an ice crystal is being imaged under an octane environment[54]. The entire AFM is housed in a nitrogen-cooled enclosure and fine-tuning of the temperature is achieved using a sample holder with an embedded peltier cooler. Ice surfaces are highly dynamic making imaging challenging even at these low temperatures. In the third example a pit is evolving in aluminum alloy (AA 6082) that has been alloyed for improved strength and corrosion resistant properties[55]. In alloyed materials, there is 
great interest in determining the location and material properties of the initial sites of pitting. In the last example, copper oxide crystals are shown nucleating under electrochemical control on a conductive substrate[56]. In this example a copper wire is threaded into the fluid cell to serve as a reference electrode, a platinum wire is threaded into the outport serving as a counter electrode, and the sample substrate is the working electrode. For faceted islands such as these, the atomic steps are too closely spaced to be resolved, however the facet velocities can be quantified to help understand shape evolution. The three-electrode electrochemical arrangement can be used to either electrodeposit or to initiate corrosion.

The next frontier for crystal growth will be dynamics and species identification on time and length scales commensurate with atomic motion. There is already some indication that aspects of these problems are coming reality with true in situ vacuum STM[15], video rate STM[57], atom tracking techniques, and true atomic resolution using non-contact AFM[58,59]. As always the trick is to combine dynamic growth with appropriate time and spatial sensitivity. The next breakthrough is likely to be an especially noteworthy one as computational and experiments walk steadily towards each other.

a
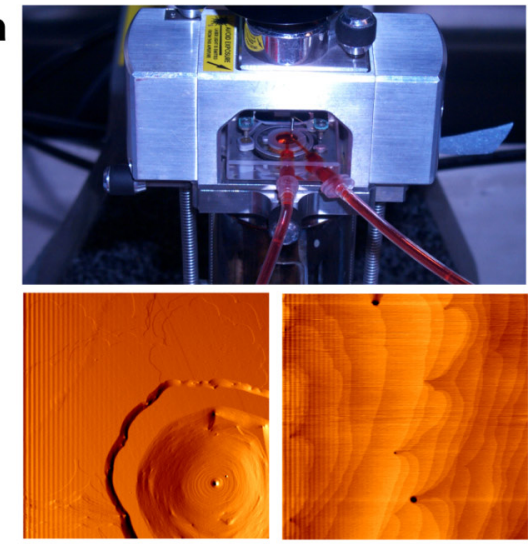

b
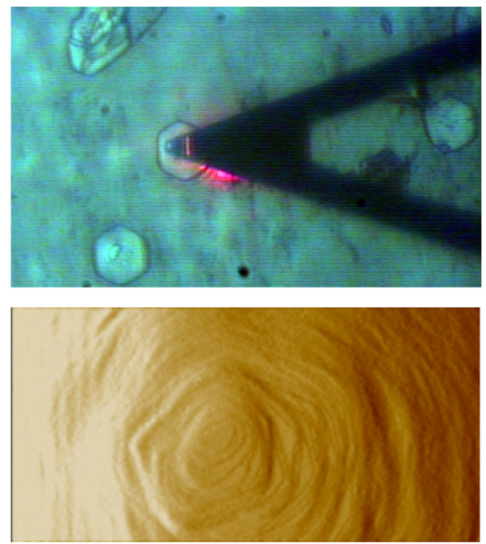

C
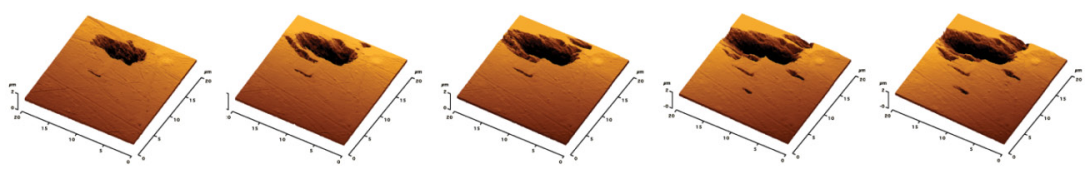

d

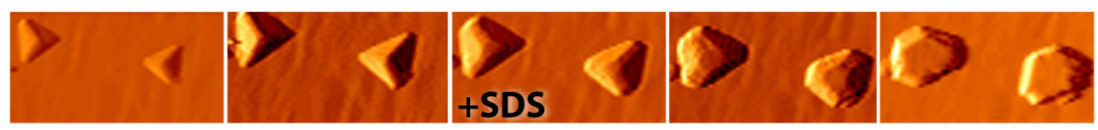

FIGURE 13. Examples of imaging in challenging environments. a) An AFM scan head with a fluorescent DMSO-based solvent highlighting the flow in and out of the fluid cell. Teflon-lined tubing is necessary for aggressive solvents such as these. Below are AFM images $(10 \mu \mathrm{mx} 10 \mu \mathrm{m})$ of the surface topography in an undersaturated (left) and supersaturated (right) solution. The transformation from etch pits to stepflow growth as the concentration of TATB increases defines the solubility. b) An optical image of the AFM cantilever above a hexagonal ice crystal at $-20 \mathrm{C}$ and the corresponding AFM images $(7.5 \mu \mathrm{mx} 15 \mu \mathrm{m})$ of a roughly hexagonal hillock showing individual ice steps at the apex and bunched steps along the sides. c) A sequence of images of a growing pit on an AA6082 surface. Each image is $20 \mu \mathrm{m} \times 20 \mu \mathrm{m} \times 2 \mu \mathrm{m}$. d) A sequence of $3 \mu \mathrm{m} \times 6 \mu \mathrm{m}$ images showing the growth of a $\mathrm{Cu}_{2} \mathrm{O}$ crystal growing in $0.02 \mathrm{MCuNO}_{3}$ solution at $0 \mathrm{~V}$ with respect to a copper reference electrode $\left(\mathrm{I} \sim-5 \mu \mathrm{A} / \mathrm{cm}^{2}\right)$. The facet expression transitions from $\{100\}-$ terminated to $\{111\}$-terminated after sodium dodecly sulphate (SDS) is added. 


\section{ACKNOWLEDGEMENT}

This work was performed under the auspices of the U.S. Department of Energy by the University of California, Lawrence Livermore National Laboratory under Contract No. W-7405-Eng-48. Portions of this work were supported by the National Institutes of Health (NIDCR DE03223) and the Laboratory Directed Research and Development Program at LLNL (06-LW-090 and 07-SI-X).

\section{REFERENCES}

1. Gibbs, J. W. The Scientific Papers of J. Willard Gibbs (Dover, New York, 1961 ).

2. Lemmlein, G. G. \& Gliki, N. V. Dokl Akad Nauk Sssr+ 94, 473-\& (1954).

3. Burton, W. K., Cabrera, N. \& Frank, F. C. Nature 163, 398-399 (1949).

4. $\quad$ Chernov, A. A. J Mater Sci-Mater El 12, 437-449 (2001).

5. Chernov, A. A. J Cryst Growth 264, 499-518 (2004).

6. Binnig, G. \& Rohrer, H. Rev Mod Phys 59, 615-625 (1987).

7. Orr, B. G., Snyder, C. W. \& Johnson, M. Rev Sci Instrum 62, 1400-1403 (1991).

8. $\quad$ Ehrlich, G. \& Hudda, F. G. J Chem Phys 44, 1039-\& (1966).

9. $\quad$ Ayrault, G. \& Ehrlich, G. J Chem Phys 60, 281-294 (1974).

10. Bauer, E. Rep Prog Phys 57, 895-938 (1994).

11. Bauer, E. Appl Surf Sci 92, 20-29 (1996).

12. Mundschau, M., Bauer, E., Telieps, W. \& Swiech, W. Surf Sci 213, 381-392 (1989).

13. Giesen, M. Prog Surf Sci 68, 1-153 (2001).

14. Jeong, H. C. \& Williams, E. D. Surf Sci Rep 34, 175-294 (1999).

15. Tsukamoto, S. \& Koguchi, N. J Cryst Growth 202, 118-123 (1999).

16. Ross, F. M., Tersoff, J., Reuter, M., Legoues, F. K. \& Tromp, R. M. Microsc Res Techniq 42, 281294 (1998).

17. Ross, F. M. Mater Today 9, 54-55 (2006).

18. Xin, R. L., Leng, Y. \& Wang, N. J Cryst Growth 289, 339-344 (2006).

19. Gratz, A. J. \& Hillner, P. E. Abstr Pap Am Chem S 203, 23-GEOC (1992).

20. Chernov, A. A., De Yoreo, J., Rashkovich, L. N. \& Vekilov, P. G. MRS Bull 29, 927-934 (2004).

21. Rashkovich, L. N., De Yoreo, J. J., Orme, C. A. \& Chernov, A. A. Crystallogr Rep+ 51, 10631074 (2006).

22. Binnig, G., Quate, C. F. \& Gerber, C. Phys Rev Lett 56, 930-933 (1986).

23. Binnig, G., Rohrer, H., Gerber, C. \& Weibel, E. Phys Rev Lett 50, 120-123 (1983).

24. Meyer, E., Hug, H. J. \& Bennewitz, R. Scanning Probe Microscopy: The Lab on a Tip (Springer, 2004) and references therein.

25. Israelachvili, J. N. Intermolecular and Surface Forces, Second Edition: With Applications to Colloidal and Biological Systems (Academic Press, 1992).

26. Bethke, C. M. Geochemical Reaction Modeling (Oxford University Press, Oxford, 1996).

27. Bethke, C. ( RockWare Inc., Golden, CO).

28. Pitzer, K. S. J Phys Chem 77, 268-277 (1973).

29. Gasperino, D., Yeckel, A., Olmsted, B. K., Ward, M. D. \& Derby, J. J. Langmuir 22, 6578-6586 (2006).

30. Teng, H. H., Dove, P. M., Orme, C. A. \& De Yoreo, J. J. Science 282, 724-727 (1998).

31. Voronkov, V. V. Sov Phys Crystallogr 18, 19 (1973).

32. Chernov, A. A., Petrova, E. \& Rashkovich, L. N. J Cryst Growth 289, 245-254 (2006).

33. Gratz, A. J., Hillner, P. E. \& Hansma, P. K. Geochim Cosmochim Ac 57, 491-495 (1993).

34. Teng, H. H., Dove, P. M. \& DeYoreo, J. J. Geochim Cosmochim Ac 63, 2507-2512 (1999).

35. Bosbach, D., Hall, C. \& Putnis, A. Chem Geol 151, 143-160 (1998).

36. Higgins, S. R., Bosbach, D., Eggleston, C. M. \& Knauss, K. G. J Phys Chem B 104, 6978-6982 (2000).

37. Onuma, K. Prog Cryst Growth Ch 52, 223-245 (2006). 
38. Vekilov, P. G., Kuznetsov, Y. G. \& Chernov, A. A. J Cryst Growth 121, 44-52 (1992).

39. Thomas, T. N., Land, T. A., Martin, T., Casey, W. H. \& DeYoreo, J. J. J Cryst Growth 260, 566579 (2004).

40. Heijna, M. C. R., van den Dungen, P. B. P., van Enckevort, W. J. P. \& Vlieg, E. Cryst Growth Des 6, 1206-1213 (2006).

41. Land, T. A. \& De Yoreo, J. J. J Cryst Growth 208, 623-637 (2000).

42. Reviakine, I., Georgiou, D. K. \& Vekilov, P. G. J Am Chem Soc 125, 11684-11693 (2003).

43. Land, T. A., DeYoreo, J. J. \& Lee, J. D. Surf Sci 384, 136-155 (1997).

44. Vekilov, P. G. \& Alexander, J. I. D. Chem Rev 100, 2061-2089 (2000).

45. MacDonald, J. C., Luo, T. J. M. \& Palmore, G. T. R. Cryst Growth Des 4, 1203-1209 (2004).

46. Sours, R. E., Zellelow, A. Z. \& Swift, J. A. J Phys Chem B 109, 9989-9995 (2005).

47. Becker, U., Risthaus, P., Bosbach, D. \& Putnis, A. Mol Simulat 28, 607-632 (2002).

48. Jordan, G. \& Astilleros, J. M. Am Mineral 91, 619-627 (2006).

49. Kaischew, R. \& Stranski, I. N. Z Phys Chem B 35, 427-432 (1937).

50. Stranski, I. N. \& Kaischew, R. Z Phys Chem B 26, 100-113 (1934).

51. Burton, W. K., Cabrera, N. \& Frank, F. C. Philos Tr R Soc S-A 243, 299-358 (1951).

52. Malkin, A. J., Land, T. A., Kuznetsov, Y. G., Mcpherson, A. \& Deyoreo, J. J. Phys Rev Lett 75 , 2778-2781 (1995).

53. Orme, C. A., Han, Y., Pagoria, P., Gash, A., Mitchell, A. \& Fried, L., unpublished.

54. Zepeda, S., Yeh, Y. \& Orme, C. A. Rev Sci Instrum 72, 4159-4163 (2001).

55. Gray, J. J. \& Orme, C. A., unpublished.

56. Gray, J. J., Du, D., Srolovitz, D., Choi, K. S. \& Orme, C. A., unpublished.

57. Magnussen, O. M., Polewska, W., Zitzler, L. \& Behm, R. J. Faraday Discuss 121, 43-52 (2002).

58. Hembacher, S., Giessibl, F. J. \& Mannhart, J. Science 305, 380-383 (2004).

59. Fukuma, T., Higgins, M. J. \& Jarvis, S. P. Phys Rev Lett 98, - (2007). 